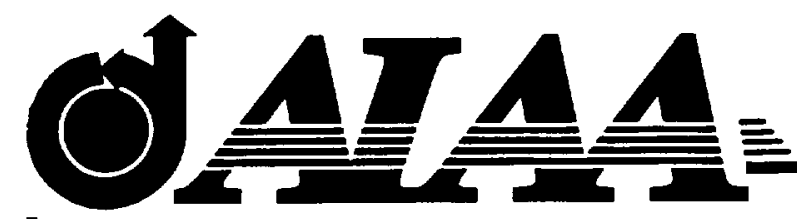

NASA-CR-202581

\title{
AIAA-96-0292
}

\section{Assessment of an Unstructured-Grid Method for Predicting 3-D Turbulent Viscous Flows}

N. T. Frink

NASA Langley Research Center Hampton, VA

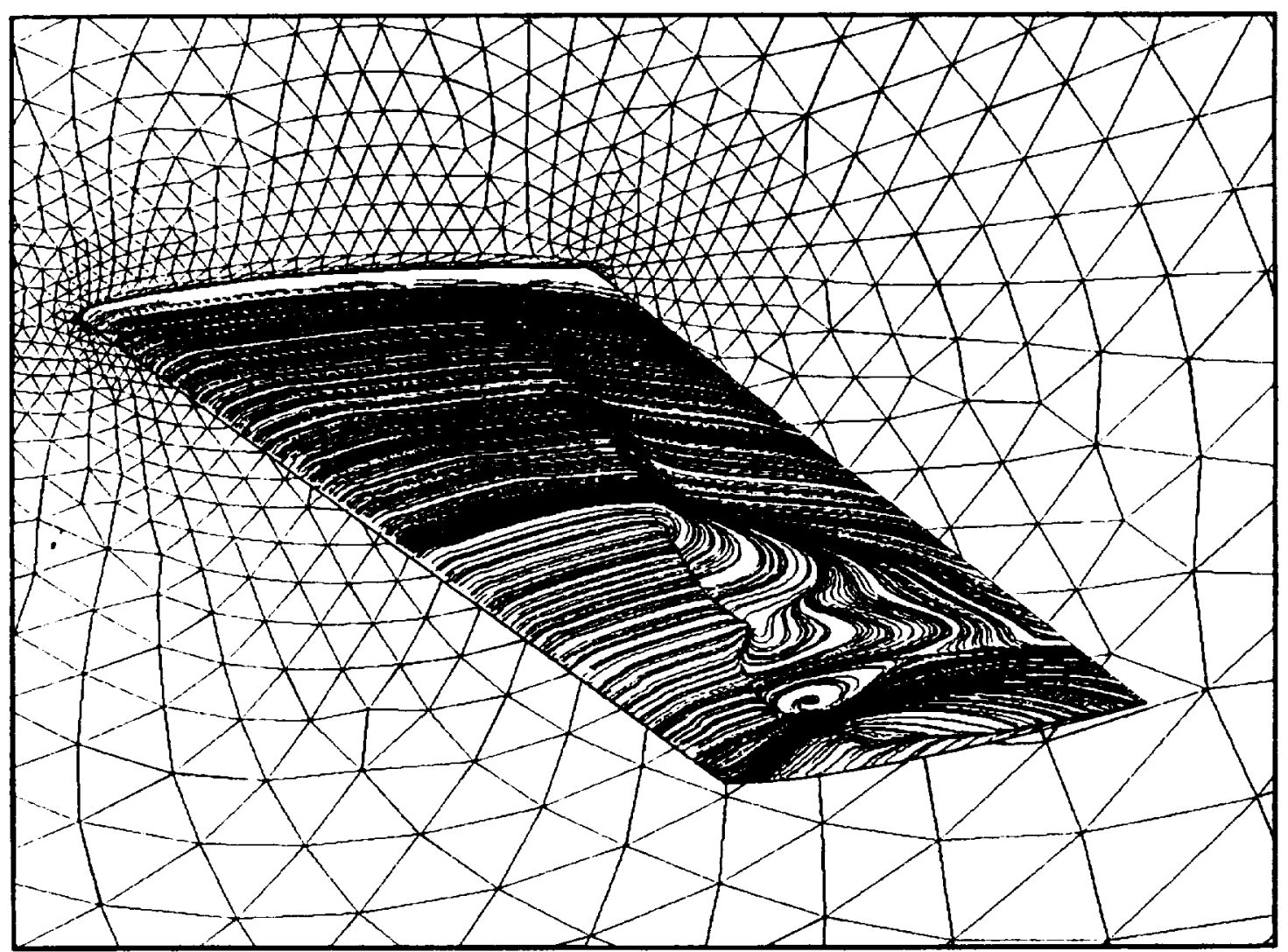

\section{4th Aerospace Sciences Meeting January 15-18, 1996/Reno, Nevada}

For permission to copy or republish, contact the American Institute of Aeronautics and Astronautics 370 L'Enfant Promenade. S.W.. Washinaton. D.C. 20024 


\title{
Assessment of an Unstructured-Grid Method for Predicting 3-D Turbulent Viscous Flows
}

\author{
Neal T. Frink* \\ NASA Langley Research Center, Hampton, Virginia
}

\begin{abstract}
A method is presented for solving turbulent now problems on three-dimensional unstructured grids. Spatial diseretization is accomplished by a cell-centered finite-volume formulation using an accurate linear reconstruction scheme and upwind flux differencing. Time is advanced by an implicit backwardEuler time-stepping scheme. Flow turbulence effects are modeled by the Spalart-Allmaras one-equation model, which is coupled with a wall function to reduce the number of cells in the sublayer region of the boundary layer. A systematic assessment of the method is presented to devise guidelines for more strategic application of the technology to complex problems. The assessment includes the accuracy in predictions of skin-friction coefficient, law-of-the-wall behavior, and surface pressure for a flat-plate turbulent boundary layer, and for the ONERA M6 wing under a high Reynolds number, transonic, separated now condition.
\end{abstract}

\section{Introduction}

Significant advancements are being made toward solving complex viscous flows on three-dimensional configurations using unstructured-grid methodology [1-8]. While solving such flows on highly-stretched tetrahedral cells is considerably more difficult than on hexahedral cells, the primary advantage is derived from the greatly reduced grid generation times. Ref. [9] has demonstrated that 'viscous' grids can be easily generated on complex shapes by the Advancing Front / Advancing Layers methodology (AFM/ALM). It is anticipated that in the near future, viscous tetrahedral grids will be generated on complex geometries in a matter of days, as are inviscid tetrahedral grids today.

The viscous, tetrahedral-based unstructured flow solution methodology is maturing along two tracks; node-centered and cell-centered schemes, each with their relative merits. Node-centered schemes exploit an efficient edge-based data structure, and have demonstrated multigrid and parallel computer implementations [2,5], but generally require large tetrahedral grids. Cell-centered schemes exploit geometric features of tetrahedra for constructing accurate spatial reconstruction schemes, and provide comparable accuracy with fewer tetrahedra, but have not been extended to multigrid or parallel architectures, and have exhibited some limitations in solution stability.

There is a need for systematic assessments of the accuracy and behavior of the various schemes. The present work focuses on an assessment of the upwind, tetrahedral cellcentered finite-volume scheme of Ref. [10]. This method is extended herein to include the Spalart-Allmaras one-equation turbulence model, and the coupling of that model with a wall function to reduce the number of cells in the sublayer region of the boundary layer. It is anticipated that the wall function approach may be applicable to 3D separated flows since flow is not stagnant along separation lines.

\section{*Senior Research Engineer, Associate Fellow AIAA}

Copyright $@ 1996$ by the American Institute of Aeronautics and Astronautics, Inc. No copyright is asserted in the United Stales under Title 17, U.S. Code. The U.S. Government has a royalty-free license to exercise all rights under the copyright claimed herein for government purposes. All other rights are reserved by the copyright owner.
The assessments will be derived from the flat-plate boundary layer problem, and the ONERA M6 wing at a high Reynolds number, transonic, separated flow condition. Key issues will be addressed related to applying a tetrahedral based, cell-centered Navier-Stokes method to turbulent-flow problems. The objectives of the study are to:

1. assess the accuracy of computing turbulent-flow pressure distributions and skin friction coefficients with tetrahedral cells,

2. investigate the accuracy and utility of a wall function formulation for computing 3-D high Reynolds number, transonic, separated flow with tetrahedral cells,

3. establish guidelines for generating unstructured, tetrahedral 'viscous' grids for solving turbulent flow problems accurately and efficiently,

4. demonstrate a mesh sequencing strategy for accelerating solution convergence.

\section{Governing Equations}

The fluid motion is governed by the time-dependent Navier-Stokes equations for an ideal gas which express the conservation of mass, momentum, and energy for a compressible Newtonian fluid in the absence of external forces. The equations are given below in integral form for a bounded domain $\Omega$ with the boundary $\partial \Omega$

$$
\frac{\partial}{\partial d} \iiint_{\Omega} \mathbf{Q d V}+\iint_{\partial \Omega} F(Q) \cdot \hat{\mathbf{n} d S}=\iint_{\partial \Omega} G(\mathbf{Q}) \cdot \hat{\mathbf{n d}} \mathrm{d} S
$$

where $Q=\left[\rho, \rho u, \rho v, \rho w, e_{0}\right]^{T}$

and

$$
F(Q) \cdot \hat{A}=(\mathbf{V} \cdot \hat{\mathbf{A}})\left[\begin{array}{c}
\rho \\
\rho \mathbf{v} \\
\rho v \\
\rho \mathbf{w} \\
\mathbf{e}_{\mathrm{o}}+\mathrm{p}
\end{array}\right]+\mathrm{p}\left[\begin{array}{c}
0 \\
\hat{n}_{\mathrm{x}} \\
\hat{n}_{y} \\
\hat{n}_{z} \\
0
\end{array}\right]
$$


and

$$
\begin{gathered}
\mathbf{G}(\mathbf{Q}) \cdot \hat{\mathbf{A}}=\frac{M_{-}}{R e_{L}}\left(n_{x} G_{1}+n_{y} G_{2}+n_{z} G_{3}\right) \\
G_{1}=\left[\begin{array}{c}
0 \\
\tau_{x x} \\
\tau_{x y} \\
\tau_{x z} \\
u \tau_{x x}+v \tau_{x y}+w \tau_{x z}-\dot{q}_{x}
\end{array}\right] \\
\mathbf{G}_{2}=\left[\begin{array}{c}
0 \\
0 \\
\tau_{x y} \\
\tau_{y y} \\
\tau_{y z} \\
+v \tau_{y y}+w \tau_{y z}-\dot{q}_{y}
\end{array}\right] \\
G_{3}=\left[\begin{array}{c}
\tau_{x z} \\
0 \\
\tau_{z z} \\
u \tau_{x z}+v \tau_{y z}+w \tau_{z z}-\dot{q}_{z}
\end{array}\right]
\end{gathered}
$$

where

$$
\begin{gathered}
\tau_{x x}=2\left(\mu+\mu_{t}\right)\left[u_{x}-\frac{1}{3}\left(u_{x}+v_{y}+w_{z}\right)\right] \\
\tau_{y y}=2\left(\mu+\mu_{t}\right)\left[v_{y}-\frac{1}{3}\left(u_{x}+v_{y}+w_{z}\right)\right] \\
\tau_{z z}=2\left(\mu+\mu_{t}\right)\left[w_{z}-\frac{1}{3}\left(u_{x}+v_{y}+w_{z}\right)\right] \\
\tau_{x y}=\left(\mu+\mu_{t}\right)\left(u_{y}+v_{x}\right), \quad \tau_{x z}=\left(\mu+\mu_{t}\right)\left(u_{z}+w_{x}\right) \\
\tau_{y z}=\left(\mu+\mu_{t}\right)\left(v_{z}+w_{y}\right) \\
\dot{q}_{x}=\frac{-1}{(\gamma-1)}\left(\frac{\mu}{\operatorname{Pr}}+\frac{\mu_{t}}{P_{r_{t}}}\right) \frac{\partial T}{\partial x}, \quad \dot{q}_{y}=\frac{-1}{(\gamma-1)}\left(\frac{\mu}{\operatorname{Pr}}+\frac{\mu_{t}}{\operatorname{Pr}_{t}}\right) \frac{\partial T}{\partial y} \\
\dot{q_{z}}=\frac{-1}{(\gamma-1)}\left(\frac{\mu}{\operatorname{Pr}_{r}}+\frac{\mu_{1}}{\operatorname{Pr}_{t}}\right) \frac{\partial T}{\partial z}
\end{gathered}
$$

The equations are nondimensionalized with freestream reference values for density $\rho_{-}$and a speed of sound $a_{\infty}$. Here $\hat{n}_{x}, \hat{n}_{y}$, and $\hat{n}_{z}$ are Cartesian components of the exterior surface unit normal $\hat{a}$ on the boundary $\partial \Omega$. The Cartesian velocity components are $u, v$, and $w$ in the $x, y$, and $z$ directions, respectively. The term $e_{o}$ is the total energy per unit volume. The Prandtl number, $\mathrm{Pr}$, is assigned a value of 0.72 , and the turbulent Prandtl number, $\mathrm{Pr}_{1}$, the value 0.9 .
Laminar viscosity $\mu$, is computed by Sutherland's law. With the ideal gas assumption, the normalized values for pressure and temperature can be expressed as

$$
p=(\gamma-1)\left(e_{0}-\frac{1}{2} \rho\left(u^{2}+v^{2}+w^{2}\right)\right)
$$

and

$$
T=n \mathbf{p} / \rho
$$

where $\gamma$ is the ratio of specific heats and is prescribed as 1.4 for air.

\section{Numerical Procedure}

A finite-volume discretization is applied to Eq. 1 which results in a consistent approximation to the conservation laws where the time rate of change of the state vector $\boldsymbol{Q}$ within the domain $\Omega$ is balanced by the net fluxes of $F$ and $\boldsymbol{G}$ across the boundary surface $\partial \Omega$. The spatial domain is divided into a finite number of tetrahedral cells, with each element serving as a computational cell. Thus, the discretized solution to Eq. 1 results in a set of volume-averaged state variables $\boldsymbol{Q}$ which are in balance with the area-averaged fluxes (inviscid and viscous) across the cell faces.

\section{Inviscid Fluxes}

Inviscid flux quantities are computed across each cell face using the Roe [11] flux-difference splitting approach (FDS), or the Van Leer [12] flux-vector splitting technique (FVS). Spatial discretization is accomplished by a novel cell reconstruction process, which is based on an analytical formulation for computing solution gradients within tetrahedral cells.

\section{Cell reconstruction scheme}

The higher-order reconstruction scheme, derived in Ref. [10] and illustrated in Fig. 1, is based on a Taylor series expansion of the cell-averaged solution to the cell face. A key

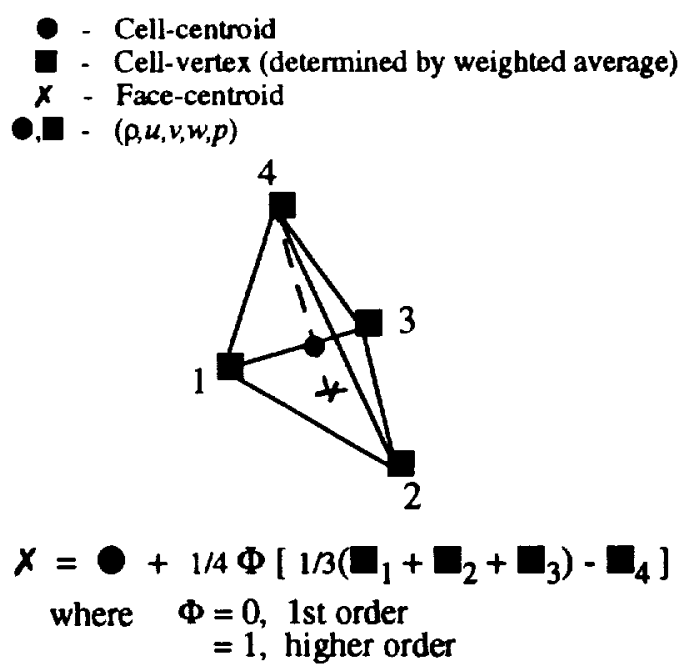

Fig. 1 Reconstruction stenctl for tetrahedral cell-centered scheme. 
component of the scheme is the reconstruction of surrounding cell-averaged data to a common vertex or node by a weighted averaging procedure. Reference [13] proposed a scheme based on an inverse-distance weighted averaging of the primitive variables from the cell centroid to the cell vertices. While this approach has proven to be both accurate and robust through wide application to inviscid problems, it is not fully second-order accurate in space. It has been shown in Ref. [14] to be approximately 1.85-order accurate.

As development efforts progressed toward solving the Navier-Stokes equations on highly stretched tetrahedral grids, it became evident that the accuracy of the inverse-distance averaging scheme was not adequate. Thus, a fully second-order accurate averaging procedure was implemented which is based on work by Holmes and Connell [4] and Rausch, et. al. [15]. The procedure is derived by solving a constrained minimization problem to determine weight factors which satisfy Laplacian relationships presented in Ref. [10]. The algorithm reconstructs to machine accuracy the exact values of a linear function at a node from surrounding cell-centered function values on an arbitrary tetrahedral grid. Furthermore, the simple universal formula shown in Fig. 1 for expanding the cell-centered data to the cell faces also reconstructs the exact value of a linear function to the cell face. Thus, the entire spatial reconstruction scheme is termed second-order accurate, which has been verified by Mitchell [14].

There is, however, an unresolved shortcoming to the Laplacian-weighted averaging scheme. Each weight factor is assumed to vary by some small perturbation from 1 . In order to achieve an exact reconstruction on highly stretched cells, these perturbations can actually become on the order of one, thus resulting in some negative weight factors. While it can be demonstrated that the computed weight factors produce an exact linear reconstruction, those with negative values violate the principle of positivity, with a detrimental impact on stability during convergence $[16,17]$. It is, thus, necessary to clip the weight factors between 0 and 2 , thereby losing some of the exactness of the linear reconstruction, but ensuring a more stable scheme.

\section{Viscous Fluxes}

The viscous fluxes $G(\boldsymbol{Q})$ are approximated at the cell-face centroids by linear reconstruction which provides a continuous representation of the solution variables across the cell faces. The stencil, presented by Mitchell [14], utilizes the averaged solution quantity at the three vertices of a cell face, $q_{-1}, q_{22}$, and $q_{23}$, and the cell-centered values of the two cells sharing the face, $q_{c 1}$ and $q_{c 2}$ where $q=(\rho, u, v, w, p)$. The derivatives for $\mathrm{u}, \mathrm{v}, \mathrm{w}$, and $\mathrm{T}$ in Eq. 1, e.g. for $u_{x}, u_{y}$, and $u_{2}$, are derived from a Cramer's rule solution to

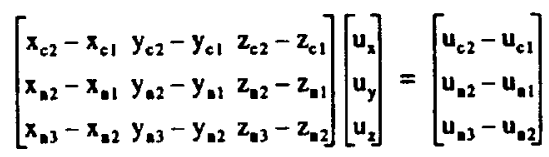

\section{Time Integration}

The viscous computations are advanced to steady state by the implicit time integration algorithm of Ref. [18]. The scheme uses the linearized, backward Euler time differencing approach to update the solution at each time step for the set of equations

$$
[A]^{n}\{\Delta Q\}^{n}=\{R\}^{n}
$$

where

$$
[A]^{\mathrm{D}}=\frac{\mathrm{V}}{\Delta \mathrm{t}} I+\frac{\partial R^{\mathrm{n}}}{\partial Q}
$$

The linear system of equations are solved at each time step with a subiterative procedure where the tetrahedral cells are grouped into "colors" (different from face-coloring) such that no two cells share a common face.

Thus, the solution is computed by solving for all the unknowns in a particular color by a point-Jacobi subiteration step before proceeding to the next color. Since the solution of the unknowns for each group can depend on those from previously computed groups, a Gauss-Seidel-like effect is realized. The method has the advantage of being completely vectorizable.

Because of the number of operations required to invert a matrix depends on the matrix bandwidth, the left-hand side of the system of linear equations is evaluated with firstorder differencing to reduce both required storage and computer time. Convergence of the subiterations is further accelerated by using Van Leer's Flux Vector Splitting (FVS) on the left-hand side. Thus in the present study, first-order differencing and FVS are applied to the left-hand side, and higher order differencing and FDS to the right-hand side. The viscous Jacobian terms are included in the left-hand side of the equation.

It is necessary to store $[A]^{\circ}$, which is a $5 \times 5$ matrix for each cell, thus, storage requirements are 180 words/cell for the implicit code. The code requires $84 \mu \mathrm{s} /$ cell/cycle on a CRAY Y-MP, or $37 \mu \mathrm{s} / \mathrm{cell} / \mathrm{cycle}$ on a CRAY C-90, with 20 subiterations and higher-order differencing. For comparison, the block-structured code CFL3D [19] requires approximately 50 words/cell and $12 \mu \mathrm{s} / \mathrm{cell} / \mathrm{cycle}$ on a Cray C-90. While there may be some room for further improvement in resource requirements of the unstructured code, such codes are typically more computer-intensive because of their generalized data structure. The success of this new technology will hinge on reducing the time and expense of generating viscous grids.

\section{Convergence acceleration}

Convergence to the steady state solution is accelerated by sacrificing the time accuracy of the scheme, and advancing the equations at each mesh point in time by the maximum permissible time step in that region. Even with such a local time stepping strategy, experience with solving 3-D viscous problems with the present cell-centered scheme has shown that maximum Courant, Friedrichs, Lewy (CFL) number is limited to approximately 25 . This limitation is a consequence of violating the principle of positivity in weighting factors, as noted an earlier section and in Refs. [16, 17].

The inherent stability limitation can be improved by scaling the CFL number according to the deviation of cell aspect ratio from the ideal value of an isotropic tetrahedron. This enables the dominate flow field to evolve quickly with the higher CFL numbers, while restricting the more temperamental 'viscous' cells. A relation has been derived 


$$
\Delta t_{\text {cort }}=\Delta t\left[1-\left(\frac{f_{\Delta t}-1}{f_{\Delta t}}\right)\left(\frac{1-\mathrm{AR}}{1-\mathrm{AR}}\right)\right]
$$

where $\quad A R=\left[9\left(V_{c}\right)^{2} /\left(S_{z_{\text {mex }}}\right)^{3}\right] / \mathrm{AR}_{\text {ideal, }}, \mathrm{AR}_{\text {iceal }}=8 /(3 \sqrt{3})$, and $f_{\Delta}$ is a scale factor. Here, $V_{c}$ is the cell volume, and $S_{f \max }$ is the area of the largest face of the cell.

The computations presented in this paper were performed with a scale factor of $f_{\Delta}=6$. Thus, for a prescribed setting of $\mathrm{CFL}=150$, the actual CFL number will be linearly scaled between 25 for the thinnest tetrahedral cell to 150 for the most isotropic cell. The ultimate benefit of this procedure was a factor-of-two reduction in required solution cycles and, hence, computer time.

\section{Turbulence Model}

\section{Spalart-Allmaras}

Closure of the Reynolds stress is provided by the oneequation Spalart-Allmaras (S-A) turbulence model [20]. This model is derived "using empiricism and arguments of dimensional analysis, Galilean invariance, and selective dependence on the molecular viscosity". The model solves a partial differential equation (PDE) over the entire field for a working variable, $\bar{v}$, from which the eddy viscosity, $\mu_{\ell}$, can be extracted. The PDE is solved separately from the flow equations using the same backward Euler time integration scheme, which results in a loosely coupled system. The production and destruction terms have been modified as recommended in Ref. [20] to ensure positive eddy viscosity throughout the computation.

On 'no-slip' surfaces, the dependent variable $\bar{v}$ is set to zero. For tangent-flow surfaces, a zero gradient of the variable is applied. Far field boundary conditions are applied by extrapolating $\bar{v}$ from the interior for outflow boundaries, and taken from the free stream for the inflow.

The S-A model requires that the distance of each cell to the nearest wall be provided for the near-wall damping terms for cells which are in proximity to 'viscous' surfaces. These distances are determined prior to code execution for cells in the "viscous" layers and contribute to only a small portion of the overall overhead.

\section{Wall Function}

The S-A model has been coupled with a wall function formulation to reduce the need for grid-resolving the flow in the sublayer portion of a turbulent boundary layer. With this approach, the inner region of the boundary layer is modeled by an analytical function which is matched with the numerical solution in the outer region. This has the advantage of 1) significantly reducing memory requirement by eliminating a large portion of cells normally required to resolve the sublayer, and 2) improving overall convergence by removing the thinner, more highly stretched cells which add stiffness to the solution process. A similar approach was successfully demonstrated in Ref. [21] where a two equation k- $\varepsilon$ turbulence model was coupled with a wall function in a modified version of the present code.

The present implementation of a wall function exploits the inherent "structure" present in viscous unstructured grids produced by the Advancing Layers Method [9]. As ev- ident in Fig. 2, the tetrahedral vertices or nodes are aligned along rays emanating from the surface.

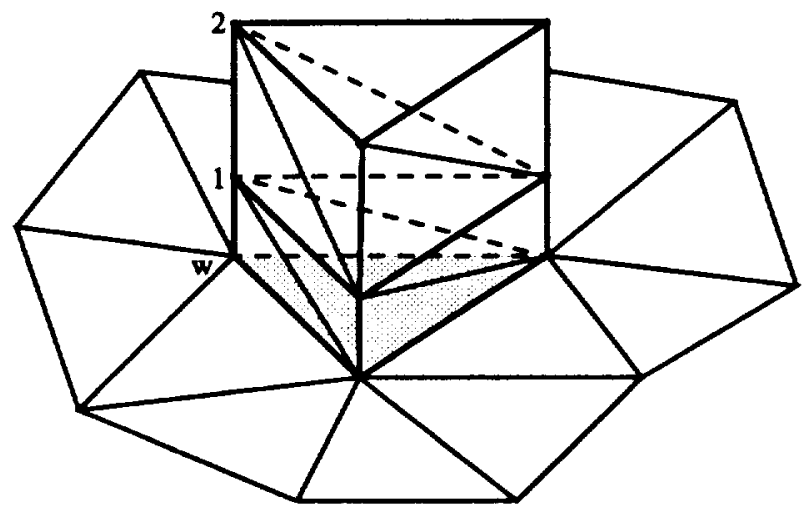

Fig. 2 Inherent "structure" of thin-layer tetrahedral grids.

The selected wall function is a law-of-the-wall expression [22] derived by Spalding in 1961 . With a single function, it models the inner laminar sublayer, a transition region, and the intermediate logarithmic layer of the turbulent boundary layer:

$$
n^{+}=u^{+}+e^{-k B}\left[e^{k u^{+}}-1-K u^{+}-\frac{\left(K u^{+}\right)^{2}}{2}-\frac{\left(K u^{+}\right)^{3}}{6}\right]
$$

where the nondimensionalized terms are

$$
\mathbf{n}^{+}=\left(\frac{R e_{L}}{M_{-}}\right) \frac{\rho_{w} n_{1} u_{*}}{\mu_{w}}, \quad u^{+}=\frac{\left|V_{1}\right|}{u_{*}}
$$

Here $\rho_{w}, \mu_{w}$ are the fluid density and laminar viscosity on the surface, respectively, and $I V, I$ the velocity magnitude at an adjacent point located a normal distance $n_{1}$ away; $u_{*}$ is the friction velocity; $\kappa=0.4$ and, $B=5.5$.

A face-centered, "slip" velocity boundary condition is determined by a two-step process. First as illustrated in Fig. 2, $\rho_{w}$ and $\mu_{w}$ are assigned values from a boundary node, and $\left|V_{1}\right|$ is defined by the reconstructed velocity magnitude at the first connected node which is located $n_{1}$ above the surface. Eq. 5 is then solved by Newton-Raphson iteration for $u_{m}$ which is assumed to apply at the boundary nodes.

Next, the computed friction velocities, $u_{a}$ from three nodes comprising a boundary face (shaded surface in Fig. 2) are averaged to establish a face-centered value, and standard face-centered flow boundary condition quantities are prescribed for $\rho_{w}$ and $\mu_{w}$. The parameter, $n_{1}$, is the normal distance to the centroid of the boundary tetrahedral cell. With these values, Eq. 5 is solved once again by Newton-Raphson iteration for the velocity magnitude, IV,I. A slip-velocity boundary condition is defined by assigning the new $\left|V_{1}\right|$ to the boundary face, and multiplying it with direction cosines extracted from a standard inviscid-type flow-tangency velocity vector.

A wall boundary condition for turbulent viscosity, which is required by the S-A PDE equation, is computed from a relation presented in Ref. [22] 


$$
\mu_{1}=\mu \kappa e^{-k \theta}\left(e^{-k u^{+}}-1-K u^{+}-\frac{\left(K u^{+}\right)^{2}}{2}\right)
$$

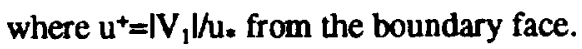

The present implementation considers no adjustment to adiabatic wall density, which is important to high speed flows. This effect will be included in future work.

\section{Results}

Results are presented below for the flat-plate boundary layer problem, and the ONERA M6 wing at high Reynolds number, transonic, separated flow conditions. The normal grid spacing across the boundary layer is prescribed by the exponential function

$$
\Delta n_{j}=\Delta n_{1}\left(1+a(1+b)^{j-1}\right)^{j-1}
$$

such that $n_{j}=n_{j-1}+\Delta n_{j-1}$.

The parameter $\Delta n_{1}$ is the spacing of the first node above the surface, while $a$ and $b$ are parameters which control the growth. An initial estimate of the normal point distribution, $\mathrm{n}^{+}$, etc., is determined by experimenting with parameter variations on an assumed $1 / 7$ th law velocity profile.

\section{Flat-Plate Boundary Layer}

The flat-plate boundary layer solution is used to assess the accuracy of the wall function in predicting flat-plate turbulent skin friction. The computations were made on quasi$2 \mathrm{D}$ unstructured grids for $\mathrm{M}_{-}=0.5, \mathrm{Re}_{\mathrm{L}}=2 \times 10^{6}$.

Grid 1 was generated by constructing a $49 \times 12$ H-topology structured grid with a normal spacing defined by $\Delta \mathrm{n}_{1}=0.001 \mathrm{~L}, a=0.3$, and $b=0.07$ in Eq. 7, which yields roughly 5 nodes across the boundary layer at $x / L=0.5$ and an approximate $\mathrm{n}^{+}$at the first node of 80 . The resulting upper domain boundary $(\mathrm{k}=12)$ is located at $0.22 \mathrm{~L}$. The $2 \mathrm{D}$ grid was stacked spanwise in $0.02 \mathrm{~L}$ increments to form three planes resulting in a 3D structured dual-channel grid (49X3X12) of H-H topology. Each hexahedral cell was subdivided into 2 prismatic cells, which were further subdivided into 3 tetrahedra each to form the 3D unstructured grid with 15,552 cells. The "flat plate" was defined by a cosine clustering between the "structured" indices $15 \leq i \leq 49$ along the $k=1$ boundary with inviscid flow prescribed on the $k=1$ boundary ahead of the plate. Boundary conditions of constant entropy and constant total enthalpy were prescribed on the inflow plane, while an extrapolation condition was applied to the upper and exit domain boundaries. A constant freestream pressure was also imposed on the exit plane.

A second grid was generated in a similar manner as the first to explore the lower limits of grid coarseness on solution accuracy. Grid 2 was constructed from a $49 \times 6$ H-topology with the Eq. 7 parameters of $\Delta n_{1}=0.001 \mathrm{~L}, a=2.0$, and $b=0.07$. This resulted in a $3 D$ channel grid (49X3X6) with 2,880 cells, and an upper domain boundary $(k=6)$ also at $0.22 \mathrm{~L}$.

Fig. 3 portrays the effect of normal grid density on the law-of-the-wall behavior at $x / L=0.5, \operatorname{Re}_{\mathrm{x}}=1 \times 10^{6}$, for the two grids. The plotted nodal solutions were reconstructed from the surrounding tetrahedral cells using the weighted averag- ing procedure discussed in an earlier section. Note that the first nodal value is matched with the log layer at approximately $\mathrm{n}^{+}=80$ for both grids. Grid 1 has 5 nodes across the boundary layer, while Grid 2 has 3 nodes.

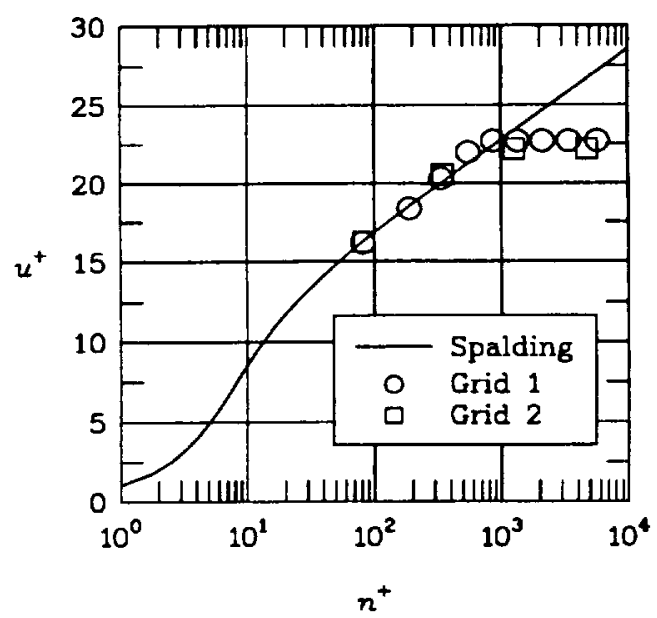

Fig. 3 Effect of grid density on law-of-the-wall behavior for fat-plate boundary layer flow, $x / L=0.5\left(M_{\infty}=0.5, R_{L}=2 \times 10^{6}\right)$.

The true test of the methodology is reflected in the skin friction coefficient in Fig. 4. Grid 1 displays excellent agreement over $0.2<x / L \leq 1.0$ with the theoretical coefficient for fully turbulent flow, $C_{p}=0.0583\left(R_{k}\right)^{-1 / 5}$, which is based on the $1 / 7$ th power law assumption. Grid 2 does not

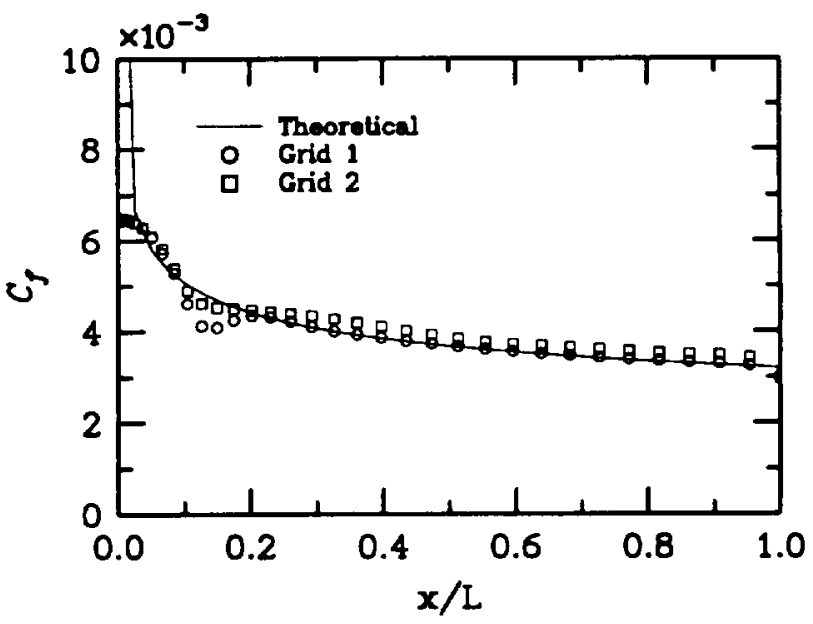

Fig. 4 Effect of grid density on skin-frictlon coefficlent for hatplate boundary layer fow $\left(M_{\infty}=0.5, R_{L}=2 \times 10^{6}\right)$.

exhibit the same level of agreement, but is remarkably close considering its extreme grid coarseness across the boundary layer in Fig. 3.

Based on experience with structured-grid computations, one would expect to need between 15 and 40 cells to adequately resolve turbulent boundary-layer flow, Thus, the results of Figs. 3 and 4 require further analysis. As noted earlier, each hexahedral cell is subdivided into 2 prismatic 
cells, which are each subdivided further into 3 tetrahedral cells. For a cell-centered scheme, each tetrahedron functions as one computational cell. Thus, a cell-averaged solution resolves the solution at three vertical positions within each prismatic cell, (for a total of six locations within the hexahedral cell). In contrast, a cell-centered structured-grid code [19], or prismatic unstructured code [23], would resolve the solution at only one vertical position within their respective cell layers. Hence in Fig. 3, there are actually 3 tetrahedral centroids between each plotted solution point which contribute to those points through the reconstruction process mentioned earlier. One can conclude from this discussion that it is more correct to consider the boundary layer as being resolved by 15 cells in Grid 1, and 9 cells in Grid 2, rather than by 5 and 3 nodes, respectively.

One final note; the spurious behavior in the computed skin friction present near the plate leading edge $(0 \leq x<0.2)$ in Fig. 4 may be due to some numerical anomalies of the weighted averaging scheme at the stagnation point where an inviscid surface suddenly changes to a viscous surface. The author plans to revisit this anomaly at a later date. The principal interest for the present study is in the fully developed turbulent flow over the remaining region of the plate.

\section{ONERA M6 Wing}

\section{Grid generation}

Tetrahedral viscous and inviscid grids were generated for the ONERA M6 wing using the VGRIDns code [9]. The VGRIDns code is based on the advancing-front method (AFM) for generating triangular surface mesh and tetrahedral volume cells.

The distribution of surface and field grid points is controlled by a 'structured' background grid [24]. This transparent grid consists of Cartesian mesh overlaying the entire domain upon which the user prescribes 'point' and 'line' sources to impose the desired spacing distribution. Parameters are available to control cell size, and the direction and intensity of spatial variation. Cells can be stretched anisotropically in directions of small gradients in order to reduce the overall grid size. A smooth variation of spacing is achieved throughout the computational domain by solving an elliptic partial differential equation on the Cartesian mesh. The approach is analogous to modeling heat diffusion from discrete heat sources in a conducting medium.

Thin-layered tetrahedra are generated in the 'viscous' regions by the advancing-layers method (ALM), which is based entirely on a modified AFM. The grid is marched away from the surface along smoothed vectors with a user prescribed distribution function, e.g. Eq. 7. As the cell sizes increase, the Cartesian background grid provides for a smooth transition to the remaining grid which is generated by the conventional AFM.

The marching process of the AlM produces prismaticlike layers of grid which are subdivided into 3 tetrahedra within each "prism", as illustrated in Fig. 2. As with the flat-plate boundary layer grids, each 'prismatic' base cell is resolved spatially by three computational cells for a tetrahedral cell-centered scheme.

\section{Test matrix}

Several tetrahedral grids, eight thin-layered and one conventional, were generated for the ONERA M6 wing (see Tables 1 and 2). The spatial sources for the background grid were prescribed to produce a coarse (6483 triangles) and a fine (8956 triangles) surface grid distribution on the wing (see Fig. 5), and remained unchanged thereafter. Anisotro-

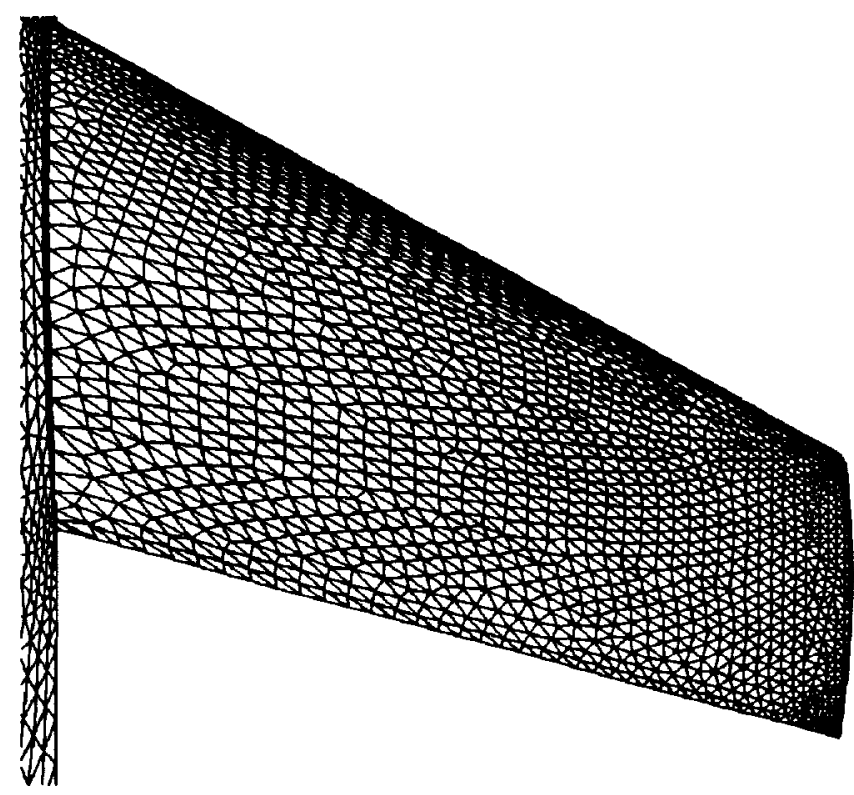

Fig. 5a Upper surface triangulation of ONERA M6 wing, coarse viscous grid, 6483 triangles on wing surface.

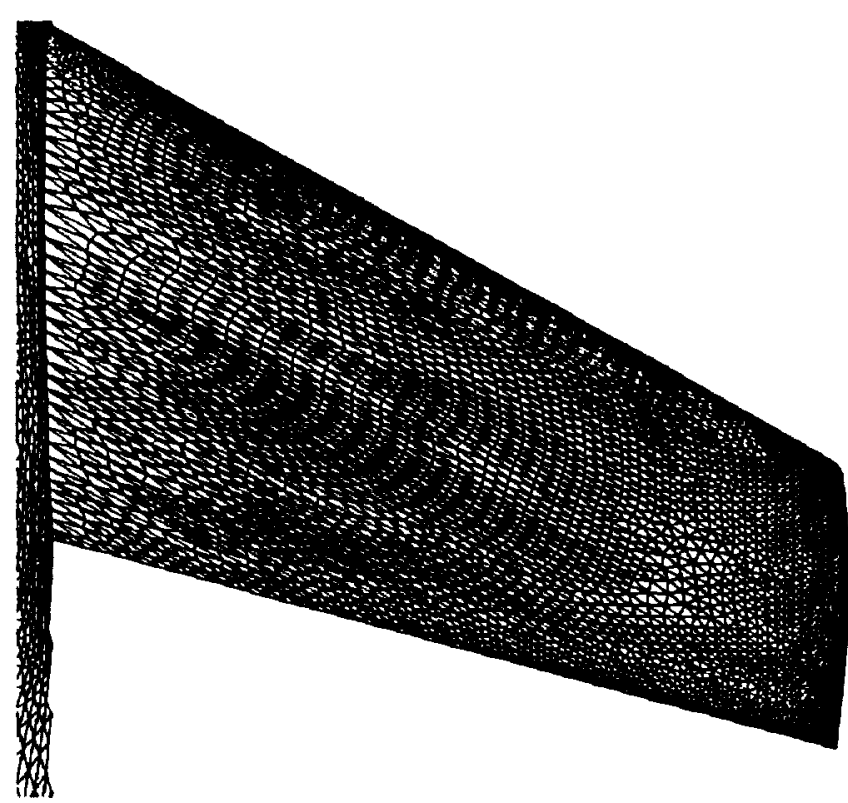

Fig. 5b Upper surface triangulatlon of ONERA M6 wing, fine viscous grid, 8956 triangles on wing surface. 
pic stretching of the surface grids was applied in the spanwise direction to reduce the total number of required cells while maintaining good chordwise resolution. A typical offbody distribution of volume grid for the coarse mesh is indirectly reflected in Fig. 6 by the centerplane grid of the WF2-

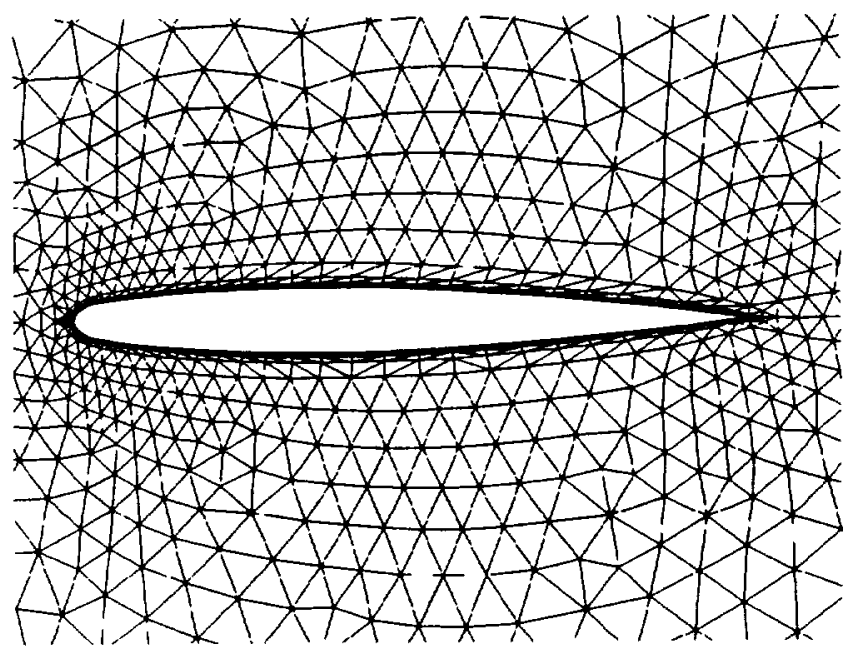

Fig. 6 Center plane triangulation of ONERA M6 wing, coarse grid, WF2-6(C).

$6(C)$ configuration. Note the smooth transition from the layered 'viscous' grid to the conventional inviscid grid. As is evident in Fig. 6, the grid has characteristics of a structured $\mathrm{O}$-mesh, since clustering of cells in the wake region has not been applied. Wake clustering is a topic for future research.

Table 1 . - Designatlons for ONERA M6 viscous tetrahedral grids

\begin{tabular}{|c|c|c|c|}
\hline \multirow{2}{*}{$\begin{array}{c}\text { Initial Spacing } \\
\left(\Delta \mathrm{n}_{1} / \mathbf{c}_{\text {ROOT }}\right) \times 10^{4}\end{array}$} & \multicolumn{3}{|c|}{$\begin{array}{c}\text { Number of cells (nodes) across boundary } \\
\text { layer }\end{array}$} \\
\hline & $\begin{array}{l}\approx 12 \text { cells } \\
\text { ( } 4 \text { nodes) }\end{array}$ & $\begin{array}{l}\approx 18 \text { cells } \\
(6 \text { nodes })\end{array}$ & $\begin{array}{l}\approx 30 \text { cells } \\
(10 \text { nodes })\end{array}$ \\
\hline 0.6 & $=$ & FV-8 ${ }^{\text {a }}$ & - \\
\hline 1.350 & - & WF1-6 & - \\
\hline 2.025 & WF2-4 & WF2-6(C,F) & WF2-10 \\
\hline 4.050 & $=$ & WF4-6 & - \\
\hline 6.075 & $=$ & WF6-6 & $=$ \\
\hline
\end{tabular}

includes two additional points in the inner layer

Table 2 . - Parameters and characterist les of viscous grids.

\begin{tabular}{|c|c|c|c|c|c|}
\hline Grid & $\underset{(\infty \infty \mathrm{Eq}, 7)}{a}$ & $\left(\begin{array}{c}b \\
(\infty \mathrm{Eq}, 7)\end{array}\right.$ & $\begin{array}{l}\text { Number } \\
\text { surface } \\
\text { triangles }\end{array}$ & $\begin{array}{c}\text { Number of } \\
\text { cells, } \\
\mathbf{N}_{\text {cell }}\end{array}$ & $\frac{\left(N_{\text {cell }}\right)_{\text {Viecoue }}}{\left(N_{\text {cell }}\right)_{\text {leviecte }}}$ \\
\hline nviscid & $\overline{=}$ & $=$ & 6483 & 258,768 & $=$ \\
\hline FV-8 & 0.5 & 0.07 & 6483 & 414,038 & 1.60 \\
\hline WF1-6 & 0.95 & 0.07 & 6483 & 356,093 & 1.38 \\
\hline WF2-4 & 2.2 & 0.00 & 6483 & 324,356 & 1.25 \\
\hline WF2-6(C) & 0.8 & 0.07 & 6483 & 356,472 & 1.38 \\
\hline WF2-10 & 0.2 & 0.07 & 6483 & 463,968 & 1.79 \\
\hline WF4-6 & 0.56 & 0.07 & 6483 & 359,268 & 1.39 \\
\hline$\overline{\text { WF6-6 }}$ & 0.432 & 0.07 & 6483 & 362,311 & 1.40 \\
\hline WF2-6(F) & 0.8 & 0.07 & 8956 & 578,556 & $=$ \\
\hline
\end{tabular}

The designations "FV" and "WF" in Tables 1 and 2 denote "full viscous" with grid resolved sublayer, and "wall function" with non-grid resolved sublayer, respectively.
The numerical nomenclature, e.g. $2-6$, provides a nominal indicator of the (initial spacing)-(number of nodes across boundary layer) at the 0.5 mean aerodynamic chord for a $R e_{\operatorname{mac}}=11.7 \times 10^{6}$.

The full viscous grid, FV-8 was designed to have approximately the same number of nodes in the outer layer of the boundary layer as the WF2-6 grid, i.e. six nodes (18 tetrahedral layers), plus two additional nodes in the sublayer, for a total of 8 nodes ( 24 tetrahedral layers).

A conventional inviscid grid was generated from the same wing surface grid, and with the same spatial source distributions as the viscous grids, thus, serving as a reference for measuring the additional cells requirements for viscous grids as shown in Table 2 . Note that the viscous grids require from 25-percent for the WF2-4 with 324,356 cells (57,490 nodes) to 79-percent for the WF2-10 with 463,968 cells $(80,927$ nodes) more tetrahedral cells than the standard inviscid unstructured grid. It is obvious from this table that grid size can become rather large if more cells are needed across the boundary layer. This factor highlights the strong need for techniques, such as a wall function, to keep the 'viscous' overhead down to manageable levels.

A structured-grid computation was repeated from Ref. [19] for comparison with the unstructured results. The grid consisted of a $193 \times 49 \times 33 \mathrm{C}-\mathrm{O}$ mesh $(294,912$ hexahedral cells) with a minimum normal spacing over the wing of $0.000015 c_{R O O T}$. This spacing matches that of the centroid of the surface tetrahedral cells in the FV-8 grid. Ref. [19] reports that this initial spacing resulted in an average $n^{+}$of 4 over the wing for $M_{\infty}=0.84, \alpha=3.06^{\circ}, \operatorname{Re}_{\text {mac }}=11.7 \times 10^{\circ}$.

\section{Solution convergence}

All turbulent flow computations in this study were performed at the flow conditions of $\mathrm{M}_{\infty}=0.8447, a=5.06^{\circ}$, and $\operatorname{Re}_{\text {max }}=11.7 \times 10^{6}$, which represents a high Reynolds number, transonic, separated-flow condition. A typical solution convergence is shown in Fig. 7.

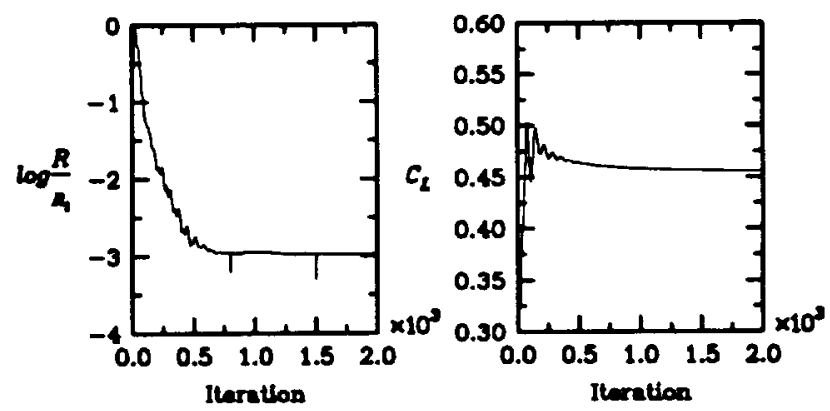

Fig. 7 Solution convergence history for WF2-6(C), $\left(M_{\infty}=0.8447, \alpha=5.06^{\circ}, \operatorname{Re}_{m a x}=11.7 \times 10^{6}\right)$

The reason for the leveling off of the residual curve at 3orders of magnitude reduction is not fully understood, but may be due to an unsteady nature of complex flow separation in the wing-tip region. Note that the lift coefficient sets up quickly, but it is necessary to run the solution longer to allow for the separated region to evolve fully.

Resource requirements for the computations are presented in Table 3 . All of the viscous cases were run with 
CFL numbers starting at 20 and ramping up to 150 over 20 cycles. The computations include the cell aspect ratio based variable CFL scaling strategy discussed earlier with a $f_{\Delta}=6$ applied. The time for the FV-8 case is based on mesh sequencing which will be described in a later section. Unresolved difficulties were encountered while attempting to start the full-viscous case from freestream initial conditions.

Table 3. - Recource requirements for unstructured cases.

\begin{tabular}{|l|c|c|c|}
\hline \hline Grid & $\begin{array}{c}\text { Number of } \\
\text { cycles }\end{array}$ & $\begin{array}{c}\text { Cray C-90 } \\
\text { time, hours }\end{array}$ & $\begin{array}{c}\text { Memory, } \\
\text { MW }\end{array}$ \\
\hline nviscid & 300 & 0.6 & 46 \\
\hline FV-8 & $900 / 425$ & $5.9^{4}$ & 75 \\
\hline WF1-6 & 1500 & 5.78 & 64 \\
\hline WF2-4 & 900 & 3.05 & 59 \\
\hline WF2-6(C) & 2000 & 7.34 & 64 \\
\hline WF2-10 & 2500 & 12.38 & 84 \\
\hline WF4-6 & 1500 & 5.75 & 65 \\
\hline WF-6 & 1500 & 5.88 & 66 \\
\hline \hline
\end{tabular}

obtained with meah sequencing

The structured-grid computation was performed with CFL3D using a multigrid strategy. The solution was converged in 500 cycles, and required $16 \mathrm{MW}$ and $0.66 \mathrm{Cray} C$ 90 hours.

\section{Comparison of methodologies}

Fig. 8 displays, the surface flow patterns for the FV-8

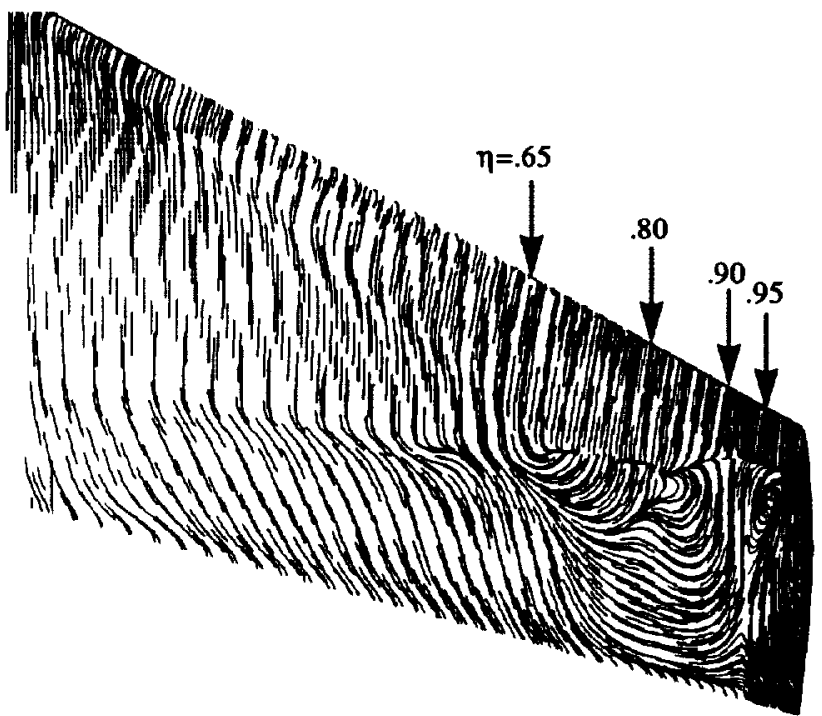

Fig. 8 Surface "oll-fow" patterns for ONERA M6 wing, FV-8 grid $\left(M_{\infty}=0.8447, \alpha=5.06^{\circ}, \operatorname{Re}_{\operatorname{mac}}=11.7 \times 10^{6}\right)$.

case, which reveals a substantial shock-induced separation on the outboard portion of the wing. These patterns were determined from the reconstructed velocities at the first node above the wing surface. The general pattern shown in Fig. 8 is representative of that from all the WF-series wall-function solutions.

Fig. 9 portrays a comparison of longitudinal $C_{p}$ distributions for unstructured full-viscous and wall function solu- tions, a full-viscous structured solution [19] obtained with the CFL3D code, and a reference unstructured inviscid result. The comparisons are presented at the four span stations

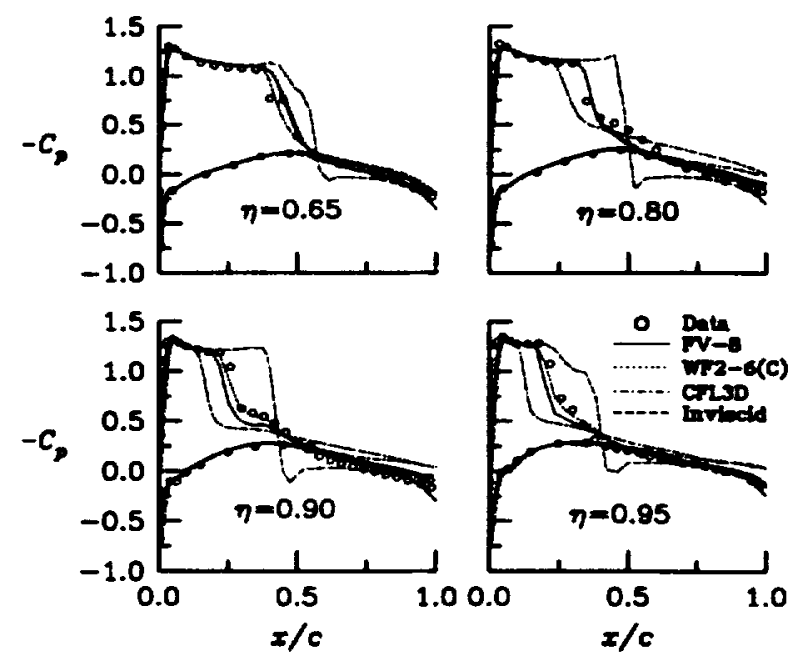

Fig. $9 C_{p}$ distributions from unstructured and structured grids for ONERA M6 wing. $\left(M_{\infty}=0.8447, \alpha=5.06^{\circ}\right.$, $\operatorname{Re}_{\operatorname{mac}}=11.7 \times 10^{6}$ ).

denoted on Fig. 8. The unstructured viscous results are in the best agreement with the experimental data of Ref. [25]. Furthermore, the wall-function solution, WF2-6(C), is in good agreement with the full-viscous solution, FV-8.

The structured result in Fig. 9, which also employs the Spalart-Almaras turbulence model, generally predicts the shock location too far forward and misses the aft-chord pressures. However, Ref. [19] demonstrated a strong dependence of the flow solution on the selection of turbulence model. Better agreement with data was shown in Ref. [19] using other turbulence models. As a note of caution, the good agreement of the unstructured results for $\eta \geq 0.90$ may be fortuitous since the flow structure at the wing tip in Fig. 8 is extremely complex and may exceed the capability of the one-equation S-A turbulence model.

\section{Surface grid sensitivity}

Fig. 10 shows the effect of surface grid density on the chordwise $C_{p}$ distributions at four chord stations. The WF2$6(C) \&(F)$ have identical initial grid spacings. The sensitivity to surface grid is small at the $\eta=0.65$ and 0.80 stations where the separation is somewhat well behaved. Differences are much larger in proximity to the more complex flow region for $\eta \geq 0.90$. The fine grid is in better agreement with the structured-grid result at the latter two stations, which once again highlights the strong sensitivities of the flow in that region. Although sensitivities to surface grid can be large in the complex tip flow region, the parametric study of normal grid spacing was performed on the coarser surface grid in order to reduce the overall computational expense. 


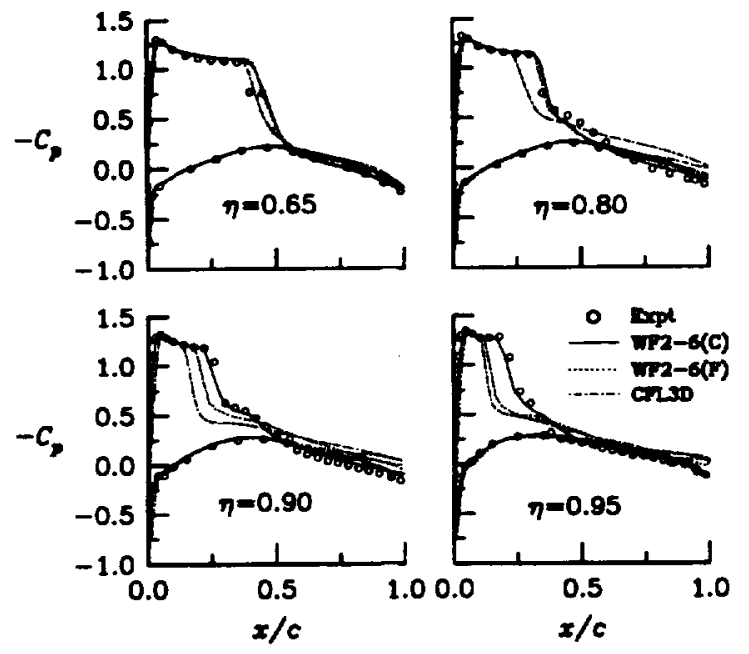

Fig. 10 Effect of surface grid density on $C_{p}$ distributions for ONERA M6 wing. $\left(M_{\infty}=0.8447, \alpha=5.06^{\circ}, \operatorname{Re}_{m a c}=11.7 \times 10^{6}\right)$.

\section{Normal grid density}

Fig. 11 depicts the effect of normal grid density (a horizontal cut across Table 1) on the chordwise $C_{p}$ distributions at four chord stations. The three solutions, which represent

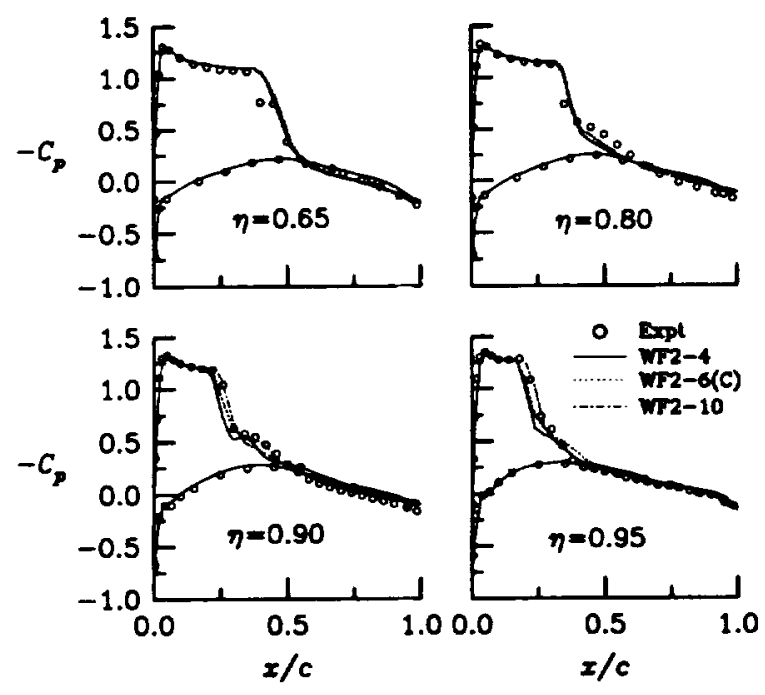

Fig. 11 Effect of normal grid density on $\mathbf{C}_{\mathrm{p}}$ distributions for ONERA M6 wing. $\left(M_{\infty}=0.8447, \alpha=5.06^{\circ}, R_{m e c}=11.7 X 10^{6}\right)$.

12-, 18-, and 30-cells across the boundary layer, are generally in good agreement with each other and the experimental data.

The law-of-the-wall behavior of the boundary layer for an attached-flow region of the wing $(x / c=0.5,2 y / b=0.15)$, is plotted in Fig. 12. The fixed initial spacing yield $n^{+}=71$ for all three cases, while there are 4,6 , and 10 nodes across the boundary layer corresponding to the WF2-4, -6 , and -10 , respectively. Recall that there are three tetrahedra between each nodal point contributing to the reconstruction of the solution to the nodes.

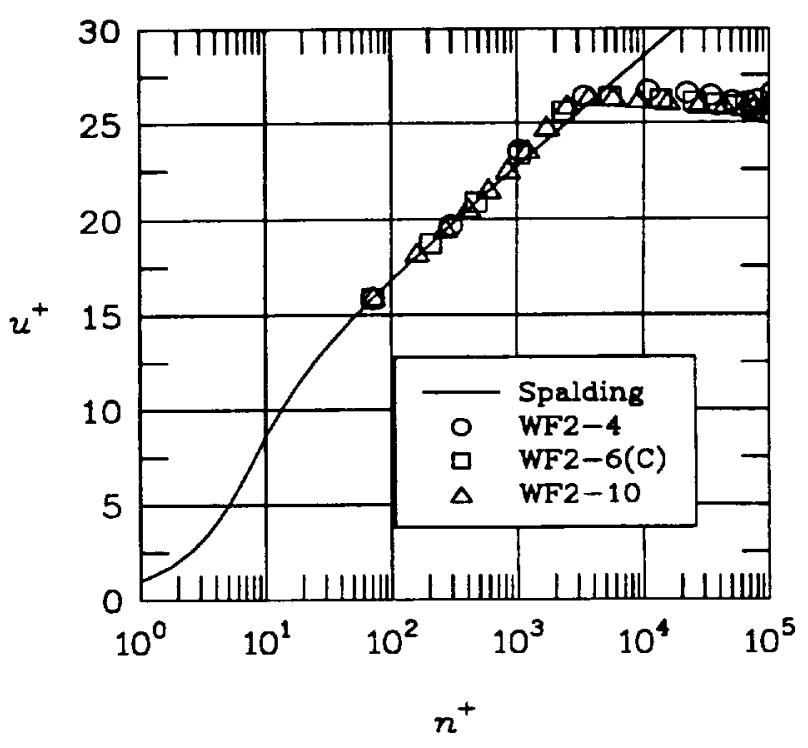

Fh. 12 Erfect of normal grid densty on law-of-the-wall behavlor at $x / c=0.5,2 y / b=0.15$ for ONERA M6 wing. $\left(M_{\infty}=0.8447\right.$, $\alpha=5.06^{\circ}, \operatorname{Re}_{\mathrm{mec}}=11.7 \times 10^{6}$ ).

\section{Effect of initial grid spacing}

Fig. 13 shows the effect of initial grid spacing (a vertical cut through the WF-series of Table 1 ) on the chordwise $C_{p}$ distributions at four chord stations. Each grid is sized to
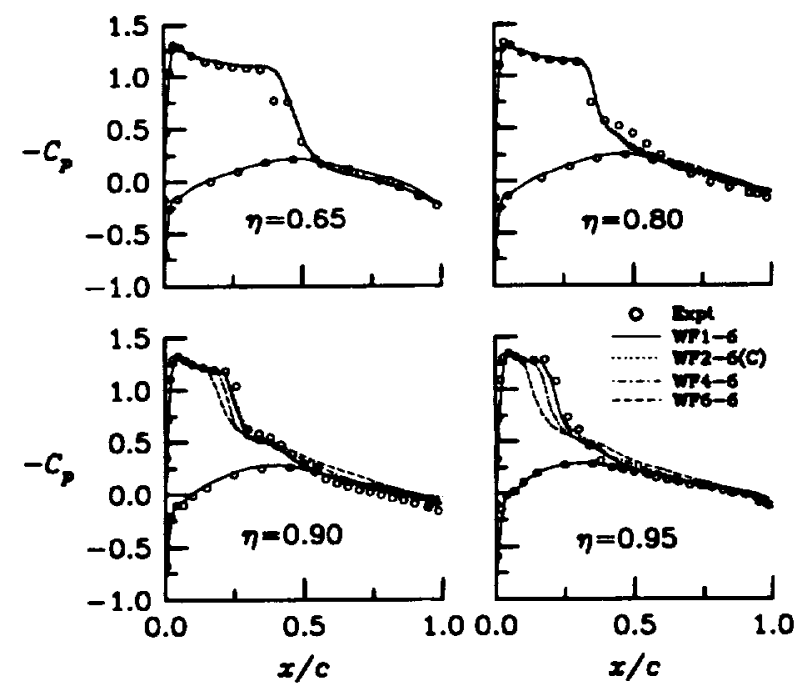

Fig. 13 Effect of initial nodal spacing on $C_{p}$ distributions for ONERA M6 wing. $\left(M_{\infty}=0.8447, \alpha=5.06^{\circ}, \operatorname{Re}_{\operatorname{mac}}=11.7 \times 10^{6}\right)$.

have approximately 6 nodes (18 tetrahedra) across the boundary layer at the midchord of the mean aerodynamic chord. For the test flow conditions, the initial grid spacings yield an $\mathrm{n}^{+}$of $48,71,146$, and 218 for the first node of the WF1-6, 2-6, 4-6, and 6-6, respectively, at $x / c=0.5,2 y /$ $\mathrm{b}=0.15$. The sensitivity to initial spacing is negligible for all cases at the $\eta=0.65$ and 0.80 stations, and for WF1- 6 and WF2-6 at $\eta=0.90$ and 0.95 . As might be expected, the agreement with data deteriorates at the higher values of $\mathrm{n}^{+}$ 
for $\eta \geq 0.90$. This result suggests that fairly large values of $n^{+}$ can be applied in conjunction with a wall function for more well behaved separated flows. However, more restricted values should be used in regions with complex 3D separated flow structures, such as spiral separations or primary saddle points, as depicted in Fig. 8.

\section{Mesh Sequencing}

The mesh sequencing strategy is often employed as a means of accelerating solution convergence. This strategy involves establishing the primary flow field relatively quickly around a configuration using a coarse mesh, then transferring that solution onto a finer mesh to complete the final grid-resolved solution.

A demonstration of this procedure is provided for two of the more costly wall function solutions from Table 3 , the WF2-6(F) and WF2-10, and for the full viscous case, the FV-8. Fig. 14 compares computer time requirements (in
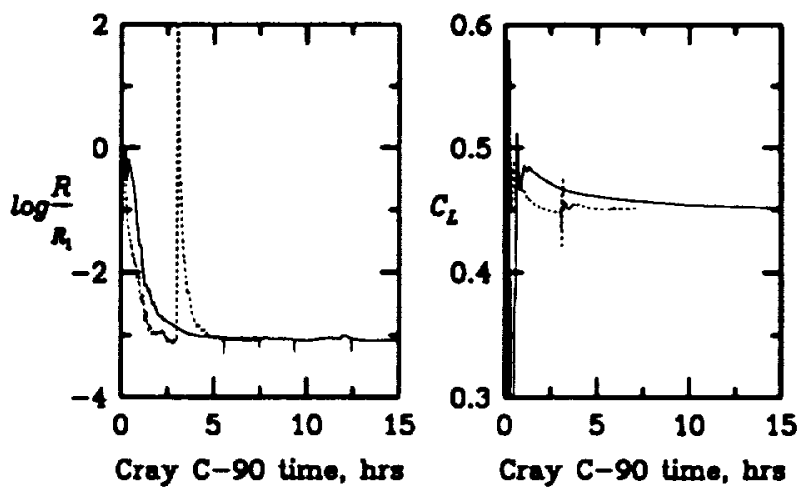

Fig. 14 Effect of mesh sequencing on solution convergence for WF2-6(F) grid. (solld - single grid solution, dash - mesh sequenced solution), $M_{\infty}=0.8447, \alpha=5.06^{\circ}, \operatorname{Re}_{\operatorname{mac}}=11.7 \times 10^{6}$.

Cray C-90 hrs) to obtain convergence of residual error and lift coefficient for the WF2-6(F) mesh. The solid curve applies to the single-grid computation which took 15.5 Cray C-90 hours for 2500 cycles. The dashed line denotes the application of mesh sequencing, starting from the coarse grid WF2-4 solution at 900 cycles (see Table 3 ), interpolating that solution onto the WF2-6(F) grid, and continuing to run for another 700 cycles with $C F L=150$. The history plots in Fig. 14 do not reflect the additional computer time used for interpolating the solution from coarse to fine mesh.

The full benefit of mesh sequencing is presented in Table 4 for the three candidate cases, which includes the overhead of interpolating solutions. Note that the total savings is on

Table 4 .- Resource requirements for mesh sequencing from caarse grid, WF2-4.

\begin{tabular}{|l|c|c|c|c|}
\hline \hline Finer grid & $\begin{array}{c}\text { C-90 brs for } \\
\text { interpolating } \\
\text { solution }\end{array}$ & $\begin{array}{c}\text { Add'1 } \\
\text { Cycles } \\
\text { beyond } \\
\text { WF2-4 }\end{array}$ & $\begin{array}{c}\text { Total } \\
\text { Solution time } \\
\text { Cray C-90 } \\
\text { hrs }\end{array}$ & $\begin{array}{c}\text { Percent } \\
\text { savings }\end{array}$ \\
\hline WF2-6(F) & 2.1 & 700 & 9.5 & 39 \\
\hline WF2-10 & 2.4 & 300 & 6.9 & 44 \\
\hline FV-8 & 1.1 & 425 & 5.9 & $\cdots$ \\
\hline \hline
\end{tabular}

the order of 40- to 45-percent for the cases shown. (A savings is not included for the FV-8 because of difficulties in obtaining a single-grid solution for that case.) An additional benefit is derived from the lower memory usage of the coarse-grid solution (59 megawords for the WF2-4), thus enabling primary flow to be setup more quickly while running in smallet queues on heavily used computers.

\section{Concluding Remarks}

A systematic study has been initiated to assess the utilization of the cell-centered unstructured scheme for obtaining accurate solutions to the Navier-Stokes equations on threedimensional configurations in an efficient manner. Closure to the flow equations is provided by a one-equation SpalartAlmaras turbulence model, which is coupled with a wall function.

Excellent accuracy in predicting the law-of-the-wall behavior and surface skin friction coefficient with tetrahedral cells was demonstrated for the flat-plate boundary layer problem. The applicability of the tetrahedral-based wall function approach to 3D high Reynolds number, transonic, separated flow was validated on a parametric set of grids for the ONERA M6 wing. The validations were supported by comparisons with experimental data and a companion structured-grid solution. The parametric study revealed that reasonable viscous solutions can be obtained with approximately 25- to 80-percent more cells, hence memory, than a standard anisotropically stretched inviscid grid. Guidelines are established for prescribing an efficient distribution of normal grid spacing. A 40- to 45-percent solution convergence acceleration was demonstrated using a mesh sequencing strategy.

While the present study concludes with useful guidelines and better understanding of the base methodology, the next step of applying this knowledge to more complex geometries is important. Work is currently underway toward that end.

\section{Acknowledgments}

The author would like to thank Dr. Shahyar Pirzadeh, ViGYAN, Inc., for generating the tetrahedral grids used in this study. The support of Dr. Paresh Parikh, Paragon Research, Inc. in performing the solution interpolations for mesh sequencing is gratefully acknowledged. The author wishes to thank Dr. Christopher L. Rumsey, Aerodynamic and Acoustic Methods Branch (AAMB) at NASA Langley Research Center (LaRC) for providing the supporting structured grid and flow solver. The consultation and advice of Dr. W. Kyle Anderson, AAMB at NASA LaRC, toward implementing the Spalart-Allmaras turbulence model is acknowledged and greatly appreciated. The computations were performed on the Numerical Aerodynamic simulation (NAS) facility and the Aeronautics Consolidated Supercomputing Facility (ACSF) at NASA Ames Research Center, and on the Cray Y-MP at NASA LaRC.

\section{References}

'Anderson, W. K., and Bonhaus, D. L.: "An implicit Upwind Algorithm for Computing Turbulent Flows on Unstructured Grids", Computers Fluids, Vol. 23, No. 1, pp. 1-21, 1994.

'Barth, T. J. and Linton, S. W.:, "An Unstructured Mesh Newton Solver for Compressible Fluid Flow and its Parallel Implementations", AlAA 95-0221, January 1995. 
${ }^{3}$ Chalot, F., Mallet, M., and Ravachol, M.: "A Comprehensive Finite Element Navier-Stokes Solver for Low- and High-Speed Aircraft Design", AIAA Paper 94-0814, January 1994.

${ }^{4}$ Holmes, D. G., and Connell, S. D., "Solution of the 2D NavierStokes Equations on Unstructured Adaptive Grids", presented at the AIAA 9th Computational Fluid Dynamics Conference, June, 1989.

'Mavriplis, D. J., “A Three Dimensional Multigrid ReynoldsAveraged Navier-Stokes Solver for Unstructured Meshes", NASA CR 194908, May, 1994.

${ }^{6}$ Marcum, D. L.; and Agarwal, R. K.: “A Three-Dimensional Finite Element Flow Solver with k- $\varepsilon$ Turbulence Model for Unstructured Grids." AIAA Paper 90-1652, 1990.

7Nakahashi, N., "FDM-FEM Zonal Approach for Viscous Flow computations Over Multiple Bodies", AlAA Paper 87-0604, January, 1987.

'Ward, S., and Kallinderis, Y.: "Hybrid Prismatic Tetrahedral Grid Generation for Complex 3-D Geometries", AIAA Paper No. 93-0669, January 1993.

Pirzadeh, S.: "Viscous Unstructured Three-Dimensional Grids by the Advancing-Layers Method", AIAA Paper No. 94-0417, January 1994.

${ }^{10}$ Frink, N. T.: "Recent Progress Toward a Three-Dimensional Unstructured Navier-Stokes Flow Solver", ALAA 94-0061, Jan. 1994.

"Roe, P. L.: Characteristic Based Schemes for the Euler Equations. Annual Review of Fluid Mechanics, Vol. 18, 1986, pp. 337 365.

${ }^{12}$ Van Leer, B.: "Flux-Vector Splitting for the Euler Equations", Eighth International Conference on Numerical Methods in Fluid Dynamics, E. Krause, ed., Volume 170 of Lecture Notes in Physics, Springer-Verlag, 1982, pp. 507-512.

${ }^{13}$ Frink, N. T.: "Upwind Scbeme for Solving the Euler Equations on Unstructured Tetrahedral Meshes." ALAA Jourmal, Vol., No. 1, January, 1992, pp. 70-77.

${ }^{14}$ Mitchell, C.R.: "Improved Reconstruction Schemes for the Navier-Stokes Equations on Unstructured Meshes", AIAA Paper 94-0642, January 1994.

${ }^{15}$ Rausch, R. D., Batina, J. T., and Yang, H. T. Y., "Spatial Adaption Procedures on Unstructured Meshes For Accurate Unsteady Aerodynamic Flow Computation." AIAA Paper 91-1106, April, 1991.

${ }^{16} \mathrm{Barth}$, T. J.: "Numerical Aspects of Computing Viscous High Reynolds Number Flows on Unstructured Meshes", AIAA Paper 91-0721, January 1991.

${ }^{17}$ Coirier, W. J.: "An Adaptively-Refined, Cartesian, Cell-Based Scheme for the Euler and Navier-Stokes Equations", NASA TM106754, October 1994.

${ }^{18}$ Anderson, W. K. "Grid Generation and Flow Solution Method for Euler Equations on Unstructured Grids", NASA TM4295, April 1992.

${ }^{19}$ Rumsey, C.L. and Vatsa, V.N.: "A Comparison of the Predictive Capabilities of Several Turbulence Models Using Upwind and Central-Difference Computer Codes", AIAA Paper 93-0192, January 1993.

${ }^{20}$ Spalart, P.R. and Allmaras, S.R.: "A One-Equation Turbulence Model for Aerodynamic Flows", AIAA Paper 92-0439, January 1992.

${ }^{21} \mathrm{~K}$ won, O. J. and Hah, C.: "Solution of the 3-D Navier-Stokes Equations with a Two-Equation Turbulence Model on Unstructured Meshes Applied to Turbomachinery", AIAA 94-1833, June 1994.

22W hite, F.M.: Viscous Fluid Flow, McGraw-Hill, Inc., ISBN O07-069710-8, 1974.

${ }^{23}$ Khawaja, A., McMorris, H., and Kallinderis, Y.: "Hybrid Grids for Viscous Flows around Complex 3-D Geometries including Multiple Bodies", AIAA 95-1685, June 1995.

${ }^{24}$ Pirzadeh, S.: "Structured Background Grids for Generation of Unstructured Grids by Advancing-Front Method", AIAA Journal, Vol. 31, No. 2, pp. 257-265, February 1993.
${ }^{25}$ Schmith, V. and Charpin, F.: "Pressure Distributions on the ONERA M6-Wing at Transonic Mach Number", AGARD Advisory Report 138, May 1979. 
